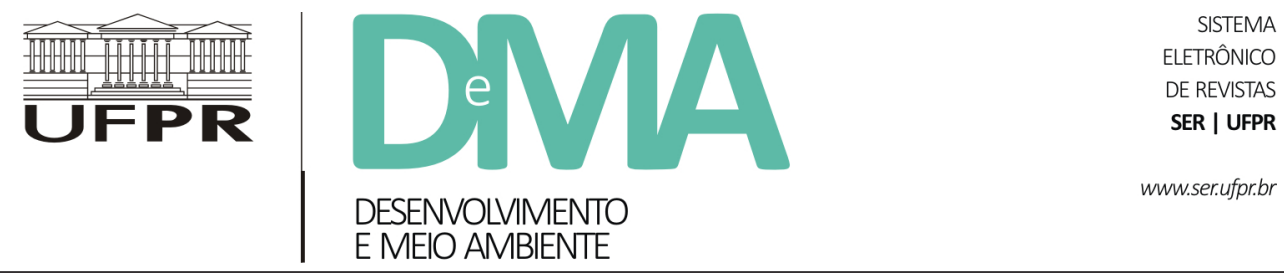

\title{
Influência das chuvas na oferta de castanha-do-brasil e o impacto no benefício socioeconômico e ambiental, no Oeste do estado do Pará
}

\section{Influence of rainfall on brazil nut supply and impact on socioeconomic and environmental benefits in the western part of the state of Pará}

\author{
Ana Karlla Magalhães NOGUEIRA ${ }^{1 *}$, Antônio Cordeiro de SANTANA ${ }^{1}$ \\ ${ }^{1}$ Universidade Federal Rural da Amazônia (UFRA), Belém, PA, Brasil. \\ *E-mail de contato: karlla_ufra@hotmail.com
}

Artigo recebido em 7 de fevereiro de 2017, versão final aceita em 9 de março de 2018.

\begin{abstract}
RESUMO: O objetivo deste trabalho foi calcular os benefícios socioeconômicos e ambientais da extração e comercialização da castanha-do-brasil (Bertholletia excelsa Bonpl.), mediante as alterações do clima na região oeste do estado do Pará, no período de 1999 a 2011, com os parâmetros estimados pelo Método Generalizado de Momentos (MGM). Os resultados mostraram que a demanda e a oferta de castanha-do-brasil são inelásticas a preço. A elasticidade cruzada indicou que o açaí comercializado no mercado paraense é complementar à castanha-do-brasil. A elasticidade-renda, com valor igual a 1,09, revelou que a castanha-do-brasil é um bem de luxo. Com relação a oferta, constatou-se que a quantidade ofertada respondeu diretamente ao preço contemporâneo da castanhado-brasil e ao preço da madeira em tora. Assim, isto indica que a madeira é um produto que não compete em terra e mão de obra com a castanha-do-brasil. Os resultados mostraram que, a partir de 1999, com as mudanças observadas no clima, houve diminuição do benefício socioeconômico ambiental para a população do oeste paraense. O benefício socioeconômico e ambiental da castanha-do-brasil atingiu o patamar de $\mathrm{R} \$ 20.842,84$ mil depois das alterações na precipitação pluviométrica regional, o que representou um decréscimo de 16,46\% em relação ao benefício obtido antes da mudança no nível das chuvas ( $\$$ \$24.951,34 mil). Com relação à distribuição dos benefícios depois das alterações no clima, consumidores foram os principais prejudicados, com uma perda de $10,22 \%$ (-R $\$ 5.406,03$ mil) dos benefícios totais.
\end{abstract}

Palavras-chave: mudança pluviométrica; castanha-do-brasil; externalidade ambiental; Região Oeste do Pará.

ABSTRACT: The objective of this study to estimate the socioeconomic and environmental benefits of Brazil nut (Bertholletia excels Bonpl.) extraction and commercialization in the period from 1999 to 2011, in the western region 
of the state of Pará, Brazil, with parameters estimatedby the Generalized Moment Method (GMM). The results showed that the demand and the supply of Brazil nuts are inelastic regarding price. The cross-elasticity indicated that the assai marketed inPará is complementary to Brazil nuts. The income elasticity, with a value equal to 1.09 , revealed that Brazil nuts a luxury commodity. Regarding the supply, it was found that the quantity offered responded directly to the contemporary price of Brazil nuts and the price of logs. This indicates that wood is a product that does not compete on land and labor with Brazil nuts. The results showed that as of 1999 , with the changes observed inrainfall, there was a reduction of the socioeconomic environmental benefit for the population of western Pará. The socioeconomic and environmental benefits of Brazil nuts reached the level of R $\$ 20,842.84$ thousand after rainfall changes in the region, which represented a decrease of $16.46 \%$ in relation to the benefit obtained before the change in rainfall levels ( $\mathrm{R} \$ 24,951.34$ thousand). Regarding the distribution of benefits after the climate change, consumers were the most affected, with a loss of $10.22 \%$ (-R\$ $5.406,03$ thousand) of total benefits.

Keywords: rainfall change; Brazil nut; environmental externality; Western region of Pará.

\section{Introdução}

A castanha-do-brasil (Bertholletia excelsa Bonpl.) é também conhecida como castanha verdadeira, castanheiro, castanha-do-pará, castanha-do-maranhão, castanheira-da-Amazônia e, no exterior, Brazil nuts, podendo medir até $60 \mathrm{~m}$ de altura e até pouco mais de $5 \mathrm{~m}$ de diâmetro (Donadio, 2002; Martins et al., 2008; Salomão, 2014). Trata-se de uma espécie encontrada em solos pobres, desestruturados, drenados e argilosos ou argilo-arenosos, adaptando-se bem em terras firmes e altas. Ela desenvolve-se em regiões de clima quente e úmido, sendo que as maiores densidades ocorrem em regiões onde predominam os tipos climáticos tropicais chuvosos, com precipitação média entre 1500 a $2800 \mathrm{~mm} / \mathrm{ano}$, e ocorrência de períodos de estiagem bem definidos (Muller et al., 1995; Martins et al., 2008).

A castanheira-do-brasil é uma das mais importantes espécies de exploração extrativista da Amazônia, tendo participação significativa na geração de divisas para a região, além de ser fonte geradora de emprego e renda para milhares de trabalhadores rurais e urbanos (Tonini, 2011; Homma, 2012; Silva et al., 2013; Bayma et al., 2014; Santana et al., 2017). A importância e dependência econômica da venda da castanha para as comunidades extrativistas, reforçam o potencial do extrativismo como uso econômico da floresta em pé na Amazônia (Nunes et al., 2011). Entretanto, Bayma et al. (2014) esclarece que os incentivos para que as comunidades rurais conservem suas florestas ocorrem apenas quando há uma perspectiva de melhoria das condições de vida destes produtores locais.

Entre os produtos florestais não madeireiros da Amazônia, a castanha-do-brasil é a mais conhecida e solidamente estabelecida no mercado doméstico, bem como, no mercado de exportação da Amazônia, principalmente, do estado do Pará, o que a torna espécie-chave para o desenvolvimento econômico-social das comunidades e para a manutenção dos benefícios diretos e indiretos da floresta (Salomão, 2009; Tonini \& Pedroso, 2014, Tonini et al., 2014). A castanha-do-brasil é considerada uma commodity, isso devido ao fato de ter o preço definido pelo mercado internacional. De acordo com o Ministério do Desenvolvimento Indústria e Comércio Exterior 
(MDIC),foram exportados (em 2015) pelo estado do Pará US\$18.181.846 em castanha-do-brasil-fresca ou seca, sem casca.

No estado do Pará, verifica-se que a coleta da castanha-do-brasil é um sistema consolidado. Portanto, representa, um polo importante do extrativismo da fruta, considerado como o principal produto florestal não madeireiro do Estado. Sua produção é sazonal, e as oscilações são decorrentes, em parte, da demanda industrial; do preço; da expansão da fronteira agrícola; da disponibilidade de mão de obra na coleta da fruta; da atuação de órgãos de controle ambiental e fiscalizadores; do esgotamento das reservas naturais; do surgimento de produtos substitutos; e, também, éfortementedependente das condições climáticas, especialmente das precipitações pluviométricas para a efetivação de produções regulares.

Rathcke \& Lacey (1985) sinalizam que a precipitação pode, de forma direta e indireta, influenciar as plantas, por afetar a produção de flores e a ação das abelhas polinizadoras. No caso específico da castanheira-do-brasil, Tonini (2011) e Santana et al. (2017) descrevem que a floração depende das condições do clima, pois a mesma é anual, longa e sincrônica, e ocorre, predominantemente, por um período médio de 6 meses durante a época de menor precipitação. Estes resultados indicam que variações significativas no nível das chuvas tende a influenciar o comportamento fenológico da castanheira, acarretando em possíveis perdas de produtividade.

Ivanov (2011), em estudo realizado em Roraima, analisou o efeito, entre outras variáveis, do clima sobre a produção de castanhais. Com base em tal estudo, concluiu que a precipitação do mês de setembro apresentou correlação positiva com a produção. Como setembro é o mês que antecede a emissão dos botões florais (Tonini, 2011), uma maior precipitação em setembro pode estimular a produção de botões florais e contribuir para o aumento da produção.

Ademais, Tonini \& Pedroso (2014) identificaram que há semelhança no padrão de produção entre populações de castanheiras relativamente distantes $(50 \mathrm{~km}$ a $150 \mathrm{~km})$ e as diferenças de produção entre os anos sugerem que fatores como as variações na distribuição das chuvas, podem ter efeito sobre o padrão de produção. Kainer et al. (2007) também observaram redução significativa na produção de árvores de castanheira-do-brasil com a redução da precipitação em anos de seca prolongada. Nesse sentido, para se obteraumento de produção da castanheira-do-brasil, é fundamental o equilíbrio na quantidade e na distribuição das chuvas nas áreas extrativistas.

Logo, a escassez e o excesso de chuvas afetam a produção, apontando para uma possível diminuição desse recurso, impondo um custo à sociedade. Esse custo pode ser compreendido como uma redução no nível de bem-estar social, decorrente da diminuição da quantidade de frutos e do aumento do preço. Portanto, reconhecendo o papel peculiar da castanheira-do-brasil no suporte ao desenvolvimento socioeconômico e ecossistêmico da região, torna-se urgente avançar nas pesquisas relacionadas com os efeitos das mudanças climáticas sobre o desenvolvimento da espécie no estado do Pará (Krag \& Santana, 2017).

A escassez e aumento da irregularidade da distribuição das chuvas tende a reduzir a oferta e, portanto, diminuir o benefício socioeconômico e ambiental produzido por esse ativo ambiental. Nesse contexto, qual a perda de benefício ou custo socioeconômico e ambiental, causada pelo excesso 
ou escassez de chuvas à produção e comercialização da castanha-do-brasil no Oeste do Pará, no período de 1999 a 2011 ?

Assim, o objetivo do trabalho foi estimar os parâmetros dos modelos de demanda e oferta de castanha-do-brasil na região Oeste do Pará, no período de 1999 a 2011. Além de calcular o valor monetário da perda do benefício socioeconômico e ambiental da produção e comercialização total de castanha (BSEATC), resultante devariação na precipitação durante o período de estudo e, também, analisar sua distribuição entre os extrativistas e consumidores. Este estudo pode fornecer informações sobre uma possível mudança na produtividade em decorrência de mudanças climáticas. Por fim, é relevante, esclarecer que o uso do termo "mudança climática" não se refere ao que tem sido discutido em termos globais, mas apenas às alterações de precipitação na área de estudo.

\section{Material e métodos}

\section{1. Área de estudo e dados utilizados}

A área de estudo é formada pelos municípios dos polos de Altamira, Itaituba e Santarém, por representarem os mercados onde é comercializada a castanha-do-brasil da região Oeste do Pará.

Os dados utilizados no trabalho são de fontes secundária, referentes as variáveis quantidades (produzidas e consumidas) de castanha-do-brasil, preços, Produto Interno Bruto (PIB) per capita (disponíveis nas estatísticas do IBGE), Índice Geral de Preços Disponibilidade Interna da Fundação Getúlio Vargas (FGV) e, por fim, a precipitação pluviométrica de estações meteorológicas do Ministério da
Agricultura, Pecuária e Abastecimento (MAPA-PA) para os polos comerciais da área de estudo, no período de 1999 a 2011. Essas séries históricas foram utilizadas na estimação dos parâmetros das equações de oferta e demanda da castanha-do-brasil, com vistas a determinar os coeficientes de elasticidades-preço, renda e cruzada.

\subsection{Métodos de Análise}

O mercado dos produtos da área pesquisada foi definido pelas equações de oferta e demanda, com base na teoria microeconômica sobre o mercado de concorrência pura, por ser a estrutura que mais se aproxima da realidade da extração, comercialização e consumo dos produtos do extrativismo.

$\mathrm{O}$ mercado de castanha-do-brasil atua com características próximas da concorrência pura pelas seguintes razões, conforme Varian (2015):

a) É um mercado em que há muitos consumidores e muitos vendedores e/ou extratores, de modo que cada um deles exerce um impacto negligenciável sobre os preços de mercado de castanha-do-brasil. Portanto, cada extrator da fruta tem um controle limitado sobre o preço, logo, os mesmos são agentes tomadores de preço;

b) A castanha-do-brasil, ofertada no mercado, é considerada um produto homogêneo na percepção dos consumidores, dado que não conseguem identificar a origem do extrativista que ofertou o produto porque não há marca ou qualidade que possa diferenciar o produto. Conforme Santana \& Khan (1992), a castanha-do-brasil não é um produto que possui marca registrada 
do estabelecimento ou da área onde a fruta é colhida, de modo que o consumidor não a identifica como produto diferenciado;

c) O mercado de castanha-do-brasil é totalmente permeável, pois não há barreiras de entrada ou de saída da atividade por novos produtores ou extratores;

d) Além disso, no mercado de castanha-do-brasil, as informações sobre os preços dos fatores de produção são de conhecimento comum dos principais agentes de mercado, tais como os consumidores e extratores.

Em conformidade com estas características, a oferta e demanda da castanha-do-brasil podem ser especificadas, na forma logarítmica, da seguinte forma (Santana, 2003; 2005):

$$
\begin{aligned}
& \ln \mathrm{QC}_{d i t}=a_{0}+a_{1} \ln \mathrm{PC}_{\mathrm{it}}+a_{2} \ln \mathrm{PAC}_{\mathrm{it}}+a_{3} \ln \mathrm{PIB}_{i t}+u_{d i t} \ldots \text { (1) } \\
& \ln \mathrm{QC}_{\text {oit }}=b_{0}+b_{1} \ln \mathrm{PC}_{\mathrm{it}}+b_{2} \ln C h u v_{i t}+b_{3} \ln \mathrm{P} M a d_{\text {it }}+u_{\text {oit }} \ldots
\end{aligned}
$$

$\ln Q C_{(d, o) \text { it }}=$ é o logaritmo natural da quantidade demandada e ofertada per capita de castanha-do-brasil, no tempo t;

$\ln P C_{\text {it }}=$ é o logaritmo natural do preço real da castanha-do-brasil, no tempo t, em $\mathrm{R} \$ / \mathrm{kg}$;

$\ln P A C_{\text {it }}=$ é o logaritmo natural do preço real do açaí, no tempo t, em $\mathrm{R} \$ / \mathrm{kg}$;

ln $P I B_{\mathrm{it}}=$ é o logaritmo natural do PIB per capita que influencia a demanda da castanha-do-brasil, no período t;

$\ln P \mathrm{Mad}_{\mathrm{it}}=$ é o logaritmo natural do preço real da madeira, no tempo t, em $\mathrm{R} \$ / \mathrm{m}^{3}$;

$\ln C h u v_{\text {it }}=$ é o logaritmo natural da variável chuva que influenciam a oferta da castanha-do-brasil no período t; $a_{\mathrm{i}} \mathrm{e} b_{\mathrm{i}}=$ são os parâmetros das equações de demanda e oferta a serem estimados $(i=1,2, \ldots, \mathrm{I}$; $n=1,2, \ldots, \mathrm{N})$;

$u_{\text {oit }} \mathrm{e} u_{\text {dit }}=$ são, respectivamente, os termos de erro das equações de oferta e demanda do produto $\mathrm{i}$, no ano t.

Na demanda, especificada pela equação 1, espera-se um sinal negativo entre a quantidade demandada $Q_{d}$ do produto i, e o preço do referido produto $P_{i}$, com sinal negativo para o parâmetro $\alpha_{i}$ conforme a Lei da Demanda (Varian, 2015). Entre as variáveis deslocadoras da demanda estão a renda per capita e o preço de produtos substitutos, cuja variação produz impacto no consumo na mesma direção. O preço de produtos complementares, é também, uma variável, cuja variação produz deslocamento da demanda, mas em sentido contrário. $\mathrm{Na}$ equação 2 , tem-se que a quantidade ofertada $Q_{o}$ do produto i é uma função direta do preço do respectivo produto $P_{i}$. Portanto, espera-se um sinal positivo para o parâmetro $\beta_{i}$, pela Lei da Oferta (Varian, 2015).

Para a estimação das equações de oferta e demanda da castanha-do-brasil, foi aplicado o Método Generalizado dos Momentos (MGM), para correção de problemas de heteroscedasticidade dos erros e autocorrelação (Santana \& Santos, 2000; Santana, 2003; Santana et al., 2011; 2012; Nogueira et al., 2013; Eviews7, 2012; Santana et al., 2017). Objetivando testar a adequação dessas variáveis à especificação do modelo, foi utilizada a estatística $J$ de Hansen (1982). Conforme Greene (2011), um valor próximo de zero indica que as variáveis instrumentais são ortogonais em relação aos erros e as equações são superidentificadas, o que atesta como correta a especificação do modelo. 
2.3. Beneficios socioeconômicos e ambientais gerados pela extração e comercialização da castanha-do-brasil

O valor monetário dos benefícios socioeconômicos e ambientais, gerados pela extração e comercialização da castanha-do-brasil das áreas de assentamento, com e sem autorização e por projetos de manejo, foi realizado com base nos fundamentos da teoria neoclássica aplicados à economia dos recursos naturais. A castanha-do-brasil tem valor de mercado e representa um dos principais produtos florestais não madeireiros do estado do Pará, por isso utilizou-se um modelo de mercado para estimar as equações de oferta (ou custo marginal social) e demanda (ou benefício marginal social) e, também, para determinar o excedente econômico ou benefício socioambiental total (Santana \& Khan, 1992; Costanza et al., 1997; El Serafy, 1998), no ano de 1999 a 2011 nas regiões da transamazônica e da BR-163 no oeste do estado do Pará.

$\mathrm{O}$ excedente econômico é uma medida do benefício marginal do consumidor, ou excedente do consumidor, dado pela diferença entre o valor que as pessoas desejam e podem pagar pelo produto e o preço efetivamente pago (área limitada entre a demanda e o preço de equilíbrio do mercado). Além do benefício marginal do produtor, ou excedente do produtor, representado pela diferença entre o preço recebido e o custo de produzir uma unidade adicional do produto (Randall \& Stoll, 1980).

Esse conceito foi, inicialmente, utilizado para determinar os retornos do investimento em pesquisa (Griliches, 1958; Peterson, 1967; Ayer \& Schuh, 1972; Lindner \& Jarret, 1978) e aplicado aos recursos naturais por Santana \& Khan (1992) e
Santana (2015), para estimar o custo social da não preservação da castanha-do-brasil. Neste trabalho, aplica-se o cálculo de integral para determinar o excedente econômico ou o benefício socioambiental da extração da castanha-do-brasil no Oeste do Pará e sua distribuição entre produtores e consumidores.

De posse dos resultados econométricos, pode-se determinar o valor do benefício socioeconômico e ambiental total do efeito das chuvas na atividade de extração e comercialização da castanha-do-brasil $\left(\right.$ BSEATCmp $\left._{t}\right)$, em tonelada, na região Oeste do Pará, dado pela soma do valor dos benefícios socioeconômicos e ambientais do produtor (BSEAP) e do consumidor (BSEAC) da castanha-do-brasilno período de 1999 a 2011.

Para obter as equações de demanda e de oferta a partir do modelo estrutural apresentado na forma logarítmica, com as quantidades apenas em função do preço, e estimar o valor do excedente econômico total ou benefício socioeconômico e ambiental da castanha-do-brasil (BSEATC), substitui-se a média de cada variável exógena nas equações de demanda e de oferta e soma-se o resultado ao intercepto de cada equação para gerar o seguinte resultado, apresentado na forma exponencial:

Demanda normal da castanha-do-brasil: $Q C d t$ $=C d P C t^{-\mathrm{c} 11}$

Oferta normal da castanha-do-brasil: $Q C o t=$ Co PCtco21

Oferta da castanha-do-brasil com a influência das mudanças nas precipitações pluviométricas: $Q C \cot =\operatorname{Cco} P C t c^{021}$

Desta forma, os parâmetros Cd, Co e Cco incorporam, respectivamente, a influência de todas as variáveis deslocadoras da demanda e da oferta, sem e com a influência das mudanças nas precipitações 
pluviométricas. O preço de equilíbrio ${ }^{1}$ da situação normal é dado por: $P C e=(C d / C o)^{1 /(\mathrm{c} 11+\mathrm{c} 21)}$ e o preço de equilíbrio da situação após o efeito da mudança nas precipitações pluviométricas é $P C c e=(C d /$ C $\mathrm{Co}^{1 /(\mathrm{c} 11+\mathrm{c} 21)}$.

Assim, o valor do BSEATC é dado pela soma da integral da equação de oferta de castanha, delimitada entre o intervalo de preço zero e o preço de equilíbrio da castanha $\mathrm{PCB}_{\mathrm{e}}$, com a integral da equação de demanda, definida no intervalo entre o $\mathrm{PCB}_{\mathrm{e}}$ e o preço em que a quantidade demandada é zero PCBd (Figura 1). O cálculo do BSEATCn $\mathrm{B}_{\mathrm{t}}$ é dado por:

$B S E A T C n_{t}=B S E A P n_{t}+B S E A C n_{t}=\int_{0}^{P C e}\left(C_{o} P C^{c 11}\right) d P C+$ $\int_{P C e}^{P C d}\left(C_{d} P C^{-c_{21}}\right) d P C$
Esse procedimento deve, também, ser feito para a situação do mercado de castanha-do-brasil com a influência adversa das alterações nas precipitações pluviométricas sobre os castanhais no período de 1999 a 2011. Assim, nominando o benefício sem a influência das mudanças no regime das chuvas de BSEATC $n_{t}$ e com seus efeitos adversos de BSEATCmp $_{\mathrm{t}}$, tem-se que o custo socioeconômico e ambiental da mudança nas precipitações pluviométricas sobre os castanhais da região Oeste do Pará (CSEACmp $)$ é dado por:

$$
\text { CSEACmp } p_{t}=\text { BSEATCmp }- \text { BSEATCn }
$$

Com isso, espera-se obter os resultados suficientes para a determinação dos benefícios socioambientais gerados pela extração de castanha-do-

\section{Quantidade (QCB)}

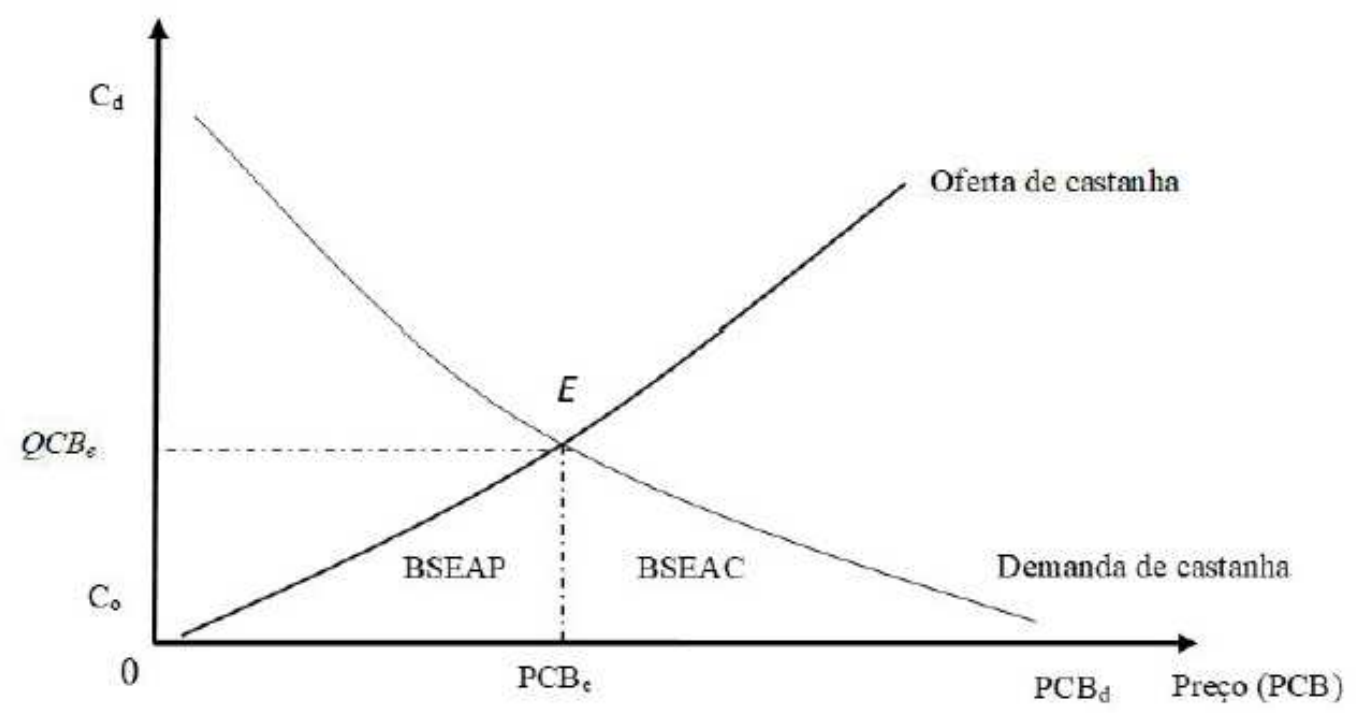

FIGURA 1 - Representação do equilíbrio de mercado da castanha-do-brasil, o excedente do produtor ou benefício socioeconômico e ambiental do produtor ou extrator (BSEAP) e o excedente do consumidor ou benefício do consumidor (BSEAC).

FONTE: Elaborado pelos autores. 
-brasil, em toneladas, na região oeste do estado do Pará, no período de 1999 a 2011.

\section{Resultados e discussão}

O modelo foi adequadamente especificado para representar o mercado de castanha-do-brasil da área de estudo, no período de 1999 a 2011. O vetor de variáveis exógenas e instrumentais foi corretamente definido, conforme atestado pela estatística $J$ de Hansen, com valor próximo de zero para o sistema de equações da Tabela 1. Essas variáveis explicaram 44,2\% e 35,2\% das variações nas quantidades demandadas e ofertadas de castanha-do-brasil. Os termos de erro não apresentaram problemas de autocorrelação de primeira ordem.
Os coeficientes de elasticidade da equação de demanda apresentaram sinais de acordo com a teoria do consumidor e significativos a 5\%. A quantidade demandada apresentou relação inversa ao comportamento dos preços, assim como o coeficiente da renda foi positivo. Portanto, incremento de $1 \%$ no preço da castanha-do-brasil e na renda do consumidor, respectivamente, tende a diminuir a quantidade demandada em $-0,61 \%$ no mesmo ano e a incrementar a renda de 1,09\%, mantendo constante a influência dos demais fatores.

Estes resultados indicam, respectivamente, que a demanda da castanha-do-brasil é inelástica a preço e que esta fruta é um bem supérfluo ou superior. Isso evidencia que, mesmo com preços elevados, a população paraense insiste em manter a

TABELA 1 - Resultados do modelo estimado de oferta e demanda de castanha-do-brasil da área de estudo, estado do Pará, 2013.

\begin{tabular}{|c|c|c|c|c|}
\hline Variável & Coeficiente & Desvio padrão & Estatística t & Probabilidade \\
\hline Constante & $-1,498020$ & 0.458421 & -3.267782 & 0.0072 \\
\hline Preço de castanha-do-brasil & $-0,611441$ & 0.136266 & -4.487104 & 0.0004 \\
\hline Preço de açaí & $-0,291641$ & 0.037789 & -7.717711 & 0.0000 \\
\hline Constante & $-2,269566$ & 2.563625 & -0.885296 & 0.3900 \\
\hline Chuva & $-0,230108$ & 0.078451 & -2.933142 & 0.0128 \\
\hline Preço de Madeira & 1,924469 & 0.432162 & 4.453120 & 0.0005 \\
\hline \multicolumn{5}{|l|}{ Demanda } \\
\hline R-squared & 0,442009 & \multicolumn{2}{|c|}{ Durbin-Watson stat } & 1.580480 \\
\hline \multicolumn{5}{|l|}{ Oferta } \\
\hline Adjusted R-squared & 0.074803 & \multicolumn{2}{|c|}{ J-statistic } & 0.333516 \\
\hline
\end{tabular}

FONTE: dados da pesquisa. 
fruta em sua dieta alimentar, devido, principalmente, as suas propriedades nutricionais.

Este resultado está em conformidade com estudo desenvolvido por Garcia (2011), realizado em mesorregiões paraenses no período de 2000 a 2009 , em que a demanda de castanha foi perfeitamente inelástica $(-0,381)$, e enquadrada como um bem superior, com elasticidade-renda igual a 1,26. Entretanto, uma situação inversa foi verificada por Santana \& Khan (1992), que estimaram a elasticidade-preço para a castanha-do-brasil em -2,631 e elasticidade-renda igual 0,717 , indicando que a fruta no estado do Pará possuía demanda elástica e enquadrando-a como um bem normal, demonstrando, respectivamente, que a preços elevados a quantidade demandada da fruta reduziria drasticamente. E, além disso, que a castanha-do-brasil é um produto essencial a dieta do consumidor e seu consumo aumenta menos que proporcionalmente ao aumento da renda.

Santana et al. (2017) estimou a elasticidade-preço da demanda da castanha-do-brasil na Amazônia (1990 a 2010) em -0,222, confirmando que a fruta é um produto inelástico a preço. O resultado estimado por Santana (2015) para a elasticidade-renda da castanha-do-brasil foi igual a 0,819 , enquadrando-o como produto essencial ou bem normal. Portanto, estando em conformidade com o resultado obtido por Santana \& Khan (1992).

A elasticidade-cruzada da demanda de castanha-do-brasil foi da ordem de -0,291, mostrando que uma variação de $1 \%$ no preço do açaí leva a variação de 2,91\% na demanda, no mesmo sentido, ceteris paribus. Este resultado indica que o açaí é um produto complementar da castanha e contraria o obtido por Garcia (2011), que obteve elasticidade-cruzada igual a 0,904 , caracterizando tais produtos como substitu- tos no consumo. Contudo, é importante acrescentar que no Oeste do Pará o consumo de açaí é totalmente diferente do resto do Pará, ficando o consumo concentrado nas famílias rurais e aos mais pobres dos centros urbanos. Naturalmente, no mercado local o consumo de açaí é pequeno e pouco disseminado no hábito da população local.

Com relação à oferta, os resultados demonstraram coerência com a teoria da produção e foram significativos a pelo menos $5 \%$, exceto o coeficiente da variável chuva que não foi significante. A quantidade ofertada respondeu diretamente ao preço contemporâneo da castanha e ao preço da madeira em tora. Assim, um aumento no preço permanente da castanha-do-brasil, de um ponto percentual, a quantidade ofertada tende a aumentar em 0,692\% no mesmo ano e a aumentar a oferta de 1,924\% em resposta a aumento no preço da madeira. Isto indica que a madeira é um produto que não compete em terra e mão de obra com a castanha-do-brasil na área de estudo.

Este resultado está em conformidade com o estudo desenvolvido por Santana (2015), que estimou a elasticidade-preço da oferta em 0,269, confirmando que a oferta da castanha-do-brasil é inelástica a preço. Para Santana (2015), esse resultado confirma a hipótese de que a castanha-do-brasil vem tornando-se mais inelástica, dado que o extrativismo está chegando ao limite da capacidade dos castanhais, cuja produção de castanha varia muito pouco em resposta aos preços de mercado, conforme propuseram Costanza et al. (1997), El Serafy (1998) e Farber et al. (2002) na formulação dos modelos de oferta para a dinâmica de exploração dos recursos naturais. Ademais, as condições climáticas influenciaram negativamente a produção de castanha-do-brasil na área de estudo. 
3.1. Análise dos resultados do benefício socioeconômico e ambiental gerados pela extração e comercialização da castanha-dobrasil

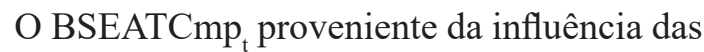
alterações das precipitações pluviométricas sobre os castanhais do oeste do Pará, tomadas a partir de 1999, foi calculado a partir das estimativas das equações de demanda normal e oferta normal de castanha-do-brasil apresentadas a seguir:

\section{Demanda Normal:}

$Q C d t=2.627,6072 \times P C t^{0,611441}$.

Oferta Normal:

$Q C o t=1.728,07037 \times P C t^{0,691773}$

Oferta com as alterações das precipitações pluviométricas:

$Q C o t=383,34913 \times P C t^{0,691773}$

Desta forma, todas as variáveis deslocadoras da demanda e da oferta sem e com a influência das alterações das precipitações pluviométricas foram incorporadas, respectivamente, nos parâmetros 2.627,6072 (Cd), 1.728,07037 (Co) 383,34913 (Cco). O preço de equilíbrio da situação normal foi obtido da seguinte forma:

$$
P C e=(2.627,6072 / 1.728,07037)^{[1 /(-0,611441+0,691773)]}
$$

e o preço de equilíbrio da situação após o efeito da mudança é:

PCce $=(2.627,6072 / 383,34913)^{[1 /(-0,611441+0,691773)]}$
Dessa forma, obteve-se preço de equilíbrio normal $(P C e)$ igual a $R \$ 1.379,285 /$ ton e quantidade de equilíbrio normal $(Q C e)$ igual a . Por outro lado, o equilíbrio com o efeito das alterações das precipitações pluviométricas $(\mathrm{PCe})$ foi igual a $R \$$ $5.449,61 /$ ton. Por fim, a quantidade de equilíbrio com o efeito das alterações das precipitações pluviométricas $(Q C e)$ igual a 931,85 ton (Figura 2).

Adotando a hipótese plausível de que o impacto de mudanças nas Precipitações Pluviométricas (IMPP) que se referem às alterações das precipitações pluviométricas na área de estudo, pode contribuir, efetivamente, para a diminuição da produção da castanheira-do-brasil no período de tempo analisado, essa diminuição da produção gera um deslocamento da curva de oferta para a direita. Dessa forma, produzindo um novo equilíbrio de mercado em que os excedentes dos consumidores e dos produtores devem diminuir e, consequentemente, o benefício socioeconômico e ambiental total para a sociedade consumidora de castanha-do-brasil.

$\mathrm{Na}$ Figura 2, é representado o mercado atual de castanha-do-brasil, com e sem os IMPP sobre sistema produtivo e/ou extrativo. A linha de oferta de cor verde representa o custo socioeconômico e ambiental dos produtores sem o IMPP, e a linha de oferta de cor azul representa o custo socioeconômico e ambiental dos produtores com o IMPP.

A área delimitada pela oferta e a esquerda do preço de equilíbrio define o excedente socioeconômico e ambiental do produtor (BSEAP). Já a área situada abaixo da demanda e a direita do preço de equilíbrio, representa o benefício socioeconômico do consumidor (BSEAC). A área abaixo da curva de oferta normal (sem alterações nas precipitações pluviométricas), a esquerda da curva de demanda e acima da linha de oferta com a influência das mu- 
danças nas precipitações pluviométricas, refere-se a área do impacto das mudanças nas precipitações pluviométricas (IMPP), ou seja, a área correspondente ao efeito das alterações das precipitações pluviométricas sobre a produção de castanha-do-brasil (Figura 2).

O benefício socioeconômico e ambiental da produção e comercialização da castanha-do-brasil, no período de 1999 a 2011, foi obtido somando-se o excedente do produtor com o excedente do consumidor. $\mathrm{O}$ valor monetário desse benefício resultou da soma da integral da equação de oferta, definida no intervalo de preço entre $R \$ 0,20 / \mathrm{kg}$ e $\mathrm{R} \$ 1,38$ / $\mathrm{kg}$, que representa o benefício socioeconômico e ambiental do produtor com o benefício socioeconô- mico e ambiental do consumidor, dado pela integral da equação de demanda, definida entre o preço de equilíbrio $\mathrm{R} \$ 1,38 / \mathrm{kg}$ e o preço $\mathrm{R} \$ 50,00 / \mathrm{kg}$, que torna a demanda aproximadamente igual a zero.

Assim, foi possível calcular os excedentes dos consumidores e produtores antes e depois dos efeitos das mudanças nas precipitações pluviométricas sobre os castanhais, por meio da aplicação do cálculo integral, e os resultados foram obtidos a seguir.

\subsubsection{Situação sem alteração no regime das chuvas}

Na situação normal, foi aplicado o cálculo integral para obter o Benefício Socioeconômico e

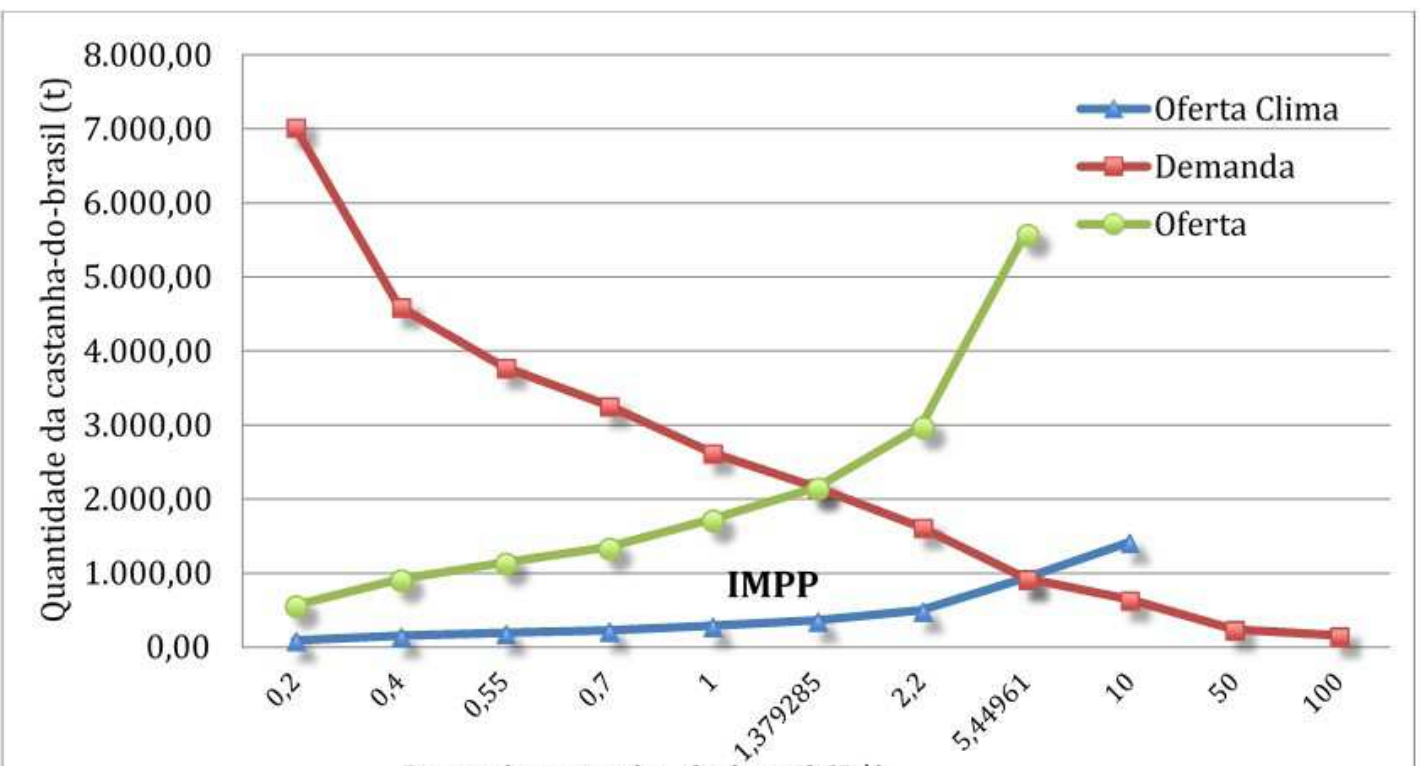

FIGURA 2 - Representação esquemáticà do excèdente do consumidór e dö prodùtor de castanha-do-brasil, considerando o efeito do Impacto das Mudanças nas Precipitações Pluviométricas (IMPP) sobre a produção da castanha no Oeste do Pará.

FONTE: Resultados da pesquisa. 


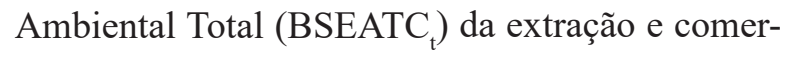
cialização da castanha-do-brasil, sem os efeitos adversos das alterações na precipitação pluviométrica regional, dado pela soma da integral do benefício socioeconômico e ambiental do produtor (BSE$\mathrm{APn}_{\mathrm{t}}$ ) o benefício socioeconômico e ambiental do consumidor $\left(\mathrm{BSEACn}_{\mathrm{t}}\right)$. Os resultados foram obtidos da seguinte forma:

BSEATCn $n_{t}=B S E A P n_{t}+B S E A C n_{t}=\int_{0,2}^{1,379285}(1.728,07$.
$\left.P C^{0,691773}\right) d P C B+\int_{1,379285}^{50,00}\left(2.627,61 . P C^{-0,611441}\right) d P C B$

BSEATCn $=\left(1.021,455 . P B C^{1,691773}\right)_{0,2}^{1,379285+}$ $\left(6.762,441 B \cdot P C B^{0,388559}\right)_{1,379285}^{50,00}$

$B S E A T C_{\mathrm{nt}}=1.692,78+23.258,56=\mathrm{R} \$ 24.951,34 \mathrm{mil} /$ ano.

Com base nos resultados, constatou-se que o BSEATC $_{t}$ gerado por ano ao longo do período de 1999 a 2011 para a população do oeste do Pará, antes do impacto das mudanças na precipitação pluviométrica regional, foi de $\mathrm{R} \$ 24.951,34 \mathrm{mil} / \mathrm{ano}$. O BSEA$\mathrm{TCn}_{\mathrm{t}}$ para os consumidores foi de $\mathrm{R} \$ 23.258,56 \mathrm{mil} /$ ano, cerca de 95,88\% dos benefícios totais. Quanto aos produtores, esses obtiveram o retorno de $\mathrm{R} \$$ $1.692,78 \mathrm{mil} / \mathrm{ano}$, o equivalente a 4,12\%. Comparativamente, os benefícios gerados aos consumidores (medido pelo excedente do consumidor) foram superiores aos benefícios dos produtores (medido pelo excedente do produtor) (Tabela 2).

\subsubsection{Situação com as alterações no regime das chuvas}

Na situação com efeito das mudanças na precipitação pluviométrica regional, aplicou-se o cálculo integral para obter o Benefício Socioeconômico e Ambiental Total BSEATCmp da extração e comercialização da castanha-do-brasil, dado pela soma da integral do benefício socioeconômico e ambiental do produtor BSEAPmp $\mathrm{t}_{\mathrm{t}}$ e o benefício socioeconômico e ambiental do consumidor BSEACmp $\mathrm{t}_{\mathrm{t}}$ Os resultados foram obtidos da seguinte forma:

$$
\begin{aligned}
& \text { BSEATCmp } t_{t}=\text { BSEAPmp }+ \text { BSEACmp }= \\
& \int_{0,2}^{5,44961}\left(383,25 . P C^{0,691773}\right) d P C B+\int_{5,44961}^{50,00}\left(2.627,61 \cdot P C^{0,611441}\right) d P C B \\
& B S E A T C m p_{\mathrm{t}}=\left(170,442 . \mathrm{PBC}^{1,691773}\right)_{0,2}^{5,44961}+ \\
& \left(6.762,441 B . P C B^{0,388559}\right)_{5,44961}^{50,00}
\end{aligned}
$$

BSEATCmp $=2.990,31+17.852,53=\mathrm{R} \$ 20.842,84$ mil/ano.

Com a mudança na precipitação pluviométrica regional, a magnitude do BSEATCmp $\mathrm{B}_{\mathrm{t}}$ anual gerado para a sociedade do Oeste do Pará foi da ordem R \$20.842,84 mil/ano. Os consumidores se apropriaram de $\mathrm{R} \$ 17.852,53 \mathrm{mil} / \mathrm{ano}$, cerca de $85,65 \%$ dos benefícios totais, obtendo uma redução de bem-estar social de, aproximadamente, 10,22\% (-R\$5.406,03 mil/ano) (Tabela 2). Quanto aos extratores, esses obtiveram a menor parcela, um montante de R\$2.990,31 mil/ano, ou seja, 14,35\% do benefício total, apresentando um aumento de bem-estar para os extratores de castanha após as alterações na precipitação pluviométrica regional.

Esse resultado decorre da mudança na elasticidade da oferta, uma vez que pelos resultados obtidos a oferta apresentou comportamento inelástico a preço e a demanda tornou-se menos inelástica após as mudanças na precipitação pluviométrica regional. Nessas condições, tem-se um cenário mais favorável aos produtores do que aos consumidores. Portanto, 
com as alterações das precipitações pluviométricas na regional BSEATCmp ${ }_{t}$ para a sociedade do Oeste Paraense apresentou uma redução de - $\mathrm{R} \$ 4.108,49$ mil, correspondendo a uma queda de bem-estar social total de $16,46 \%$ para a sociedade como um todo (Tabela 2).

$C S E A C m p_{\mathrm{t}}=B S E A T C m p_{\mathrm{t}}-B S E A T C n_{\mathrm{t}}=\mathrm{R} \$$ $20.842,84-\mathrm{R} \$ 24.951,34=-\mathrm{R} \$ 4.108,49 /$ ano

\subsubsection{Dinâmica no ajuste do bem-estar da sociedade}

A partir da análise dos resultados, constatou-se que a demanda é inelástica a preço e, nessa situação, o incremento de preço gerado pela redução da oferta aumenta os gastos dos consumidores da castanha-do-brasil, o que resulta na compra de uma quantidade menor do produto. Isto contribui diretamente na redução do bem-estar, representado pela queda média no excedente do consumidor de - R \$ 5.406,03 mil/ ano, representando diminuição de 10,22\% (Tabela 2). Logo, durante o período analisado, constatou-se que a as alterações na precipitação pluviométrica regional geraram um custo social à população paraense, suportado, principalmente, pelos consumidores da castanha-do-brasil. Isso decorre da menor oferta do fruto nos últimos anos, o que, por sua vez, contribui para o aumento do preço da fruta no mercado paraense.

Como a oferta também é inelástica, o efeito das alterações das precipitações pluviométricas produziu uma diminuição da oferta, produzindo um aumento de preço de equilíbrio de mercado de castanha-do-brasil na região do Oeste do Pará. Este cenário causa um aumento na receita líquida do extrativista da fruta, que se manifesta em melhoria do seu bem-estar, comparativamente, a situação de normalidade do clima. Este efeito produziu uma variação média no excedente do produtor de $\mathrm{R} \$ 1.297,53 \mathrm{mil} / \mathrm{ano}$, equivalente a $14,35 \%$ (Tabela 2 ). Logo, percebe-se que houve um incremento de bem-estar social dos extrativistas de castanha-do-brasil.

Portanto, tem-se que o resultado desta redistribuição de renda após as mudanças na precipitação pluviométrica regional entre os extrativistas e os consumidores da castanha-do-brasil da região Oeste do Pará, foi de -R \$ 4.108,49 mil/ano, ou queda média de $16,46 \%$ de bem-estar social ao longo do período da análise (Tabela 2).

TABELA 2 - Resultados da análise dos benefícios socioeconômicos gerados anualmente antes e depois das alterações na precipitação pluviométrica regional sobre o sistema de produção da castanha-do-brasil no estado do Pará - 1999-2011.

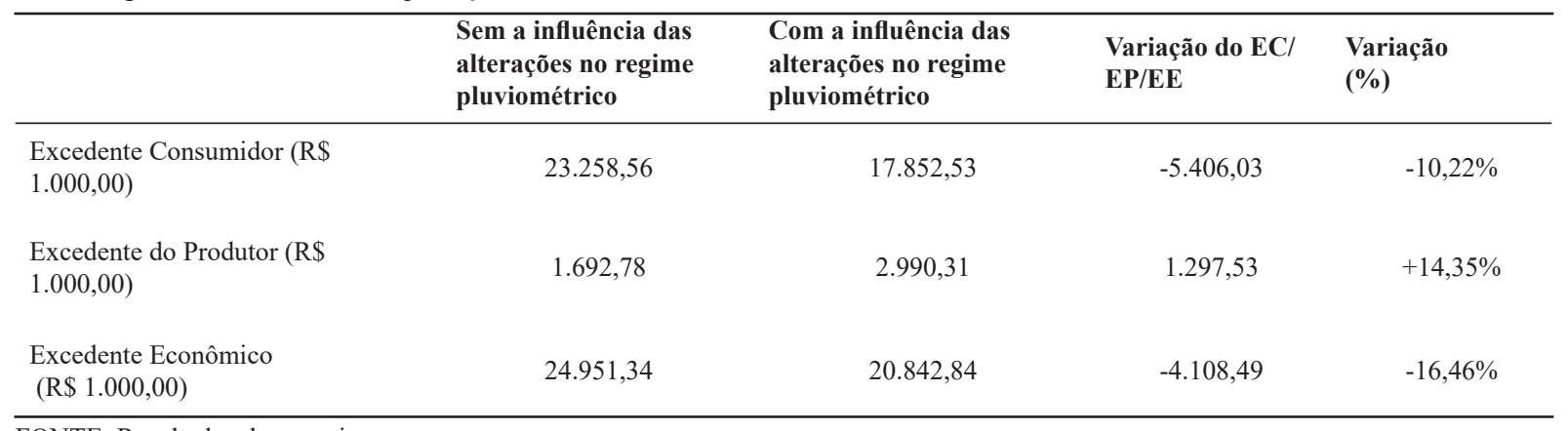

FONTE: Resultados da pesquisa. 


\section{Conclusões}

No período analisado, constatou-se que a demanda e a oferta de castanha-do-brasil foram inelásticas a preço. A elasticidade renda da demanda caracterizou a castanha-do-brasil como um bem superior. A elasticidade cruzada da demanda indicou que o açaí é um produto complementar no consumo da castanha-do-brasil. Com relação à oferta, constatou-se que quantidade ofertada aumentou $0,692 \%$ e 1,924\% quando houve, respectivamente, aumento no preço dacastanha-do-brasil e da madeira. Logo, a madeira não compete em terra e mão de obra com a castanha-do-brasil. Ademais, as variações no nível pluviométrico na área de estudo influenciaram, negativamente, a produção de castanha-do-brasil.

Finalmente, com relação a avaliação do benefício socioeconômico ambiental total, constatou-se que com a alteração das precipitações pluviométricas no sistema de produção de castanha-do-brasil, houve redução do BSEATCmp $p_{t}$ de $16,46 \%$ em relação ao benefício obtido antes da mudança climática (R\$ 24.951,34 mil). Com as mudanças nas precipitações pluviométricas, o bem-estar dos consumidores diminuiu $10,22 \%$, tendo perda de $\mathrm{R} \$ 5.406,03 \mathrm{mil} /$ ano. Houve um aumento de bem-estar para os produtores de castanha com as mudanças nas precipitações pluviométricas. Entretanto, os custos sociais oriundos dessas mudanças como uma decorrência do desmatamento e queimadas na região, afetaram negativamente toda a população com perda anual de bem-estar social no valor de -R \$ 4.108,49 mil/ano.

Logo, conclui-se que toda a população perde com as mudanças no regime das chuvas pela redução na produção e comercialização da castanha-do-brasil na Região do Oeste do Pará. Ou seja, a mudança na precipitação afetou o extrativismo da castanha-do-brasil na área de estudo, desencadeando problemas de ordem social, econômica e ambiental para uma parcela significante da população paraense. Nesse sentido, percebe-se a necessidade de minimizar os impactos das mudanças climáticas por meio do desenvolvimento de políticas públicas que busquem regular as atividades predatórias que contribuem para as alterações ocorridas no ecossistema das florestas do estado do Pará.

\section{Referências}

Ayer, H. W.; Schuh, E. Social rates of return and other aspects of agricultural research in São Paulo, Brazil. American Journal of Agricultural Economics, 54(3), 557-569, 1972.

Bayma, M. M. A.; Malavazi, F. W.; Sá, C. P. de.; Fonseca, F. L.; Andrade, E. P.; Wadt, L. H. O. Aspectos da cadeia produtiva da castanha-do-brasil no estado do Acre, Brasil. Boletim Museu Paraense Emílio Goeldi, Ciências naturais, 9(2), 417-426, 2014.

Costanza, R.; D'arge, R.; de Groot, R.; Farber, S.; Grasso, M.; Hannon, B.; Limburg, K.; Naeem, S.; O'neill, R. V.; Paruedo, J.; Raskin, R. G.; Sutton, P.; Van den belt, M. The value of the world's ecosystem services and natural capital. Nature, 15(2), 253-260, 1997.

Donadio, L. C.; Môro, F. V.; Servidone, A. A. Frutas nativas. São Paulo: Novos Talentos, 2002.

El Serafy, S. Pricing the invaluable: the value of the word's ecosystem services and natural capital. Ecological Economics, 25(1), 25-27, 1998.

Eviews7. User's Guide II. Irvine-CA: QMS, 2012. 818p.

Farber, S. C.; Costanza, R.; Wilson, M. A. Economic and ecological concepts for valuing ecosystem services. Ecological Economics, 41(2), 374-392, 2002.

Garcia, W. S. O Mercado de produtos florestais não madeireiros no Estado do Pará: açaí e castanha do Pará. 
Dissertação de Mestrado (Mestrado em Ciências Florestais). Universidade Federal Rural da Amazônia, Belém do Pará. 2011, 73p.

Greene, W. H. Econometric analysis. New Jersey: Prentice Hall, 2011. 1075p.

Griliches, Z. Research costs and social returns: hybrid corn and related innovations. Journal of Political Economy, 66(3), 419-431, 1958.

Hansen, L. P. Large sample properties of generalized method of moments estimators. Econometrica, 50(4), 1029-1054, 1982.

Homma, A. K. O. Extrativismo vegetal ou plantio: qual a opção para a Amazônia? Estudos avançados, 26(74), 167-186, 2012. doi: 10.1590/S0103- 40142012000100012

Ivanov, G. B. Influência de variáveis dendrométricas, anatômicas e ambientais na produção de frutos e sementes de Bertholletia excelsa H.B.K. 2011. 96f. Dissertação (Mestrado em Ciências Florestais) - Universidade Federal de Santa Maria, Santa Maria, 2011.

Kainer, K. A.; Wadt, L. H. O.; Staudhammer, C. L. Explaining variation in Brazil nut fruit production. Forest Ecology and Management, 250, 244-255, 2007.

Krag, M. N.; Santana, A. C. de. A cadeia produtiva da castanha-do-brasil na região da Calha Norte, Pará, Brasil. Boletim do Museu Paraense Emilio Goeldi, Ciências Naturais, 12, 363-386, 2017. Disponível em: https://issuu.com/ bgoeldi_cn/docs/naturais_v12n3.

Lindner, R. K.; Jarret, F. G. Supply shifts and the size of research benefits. American Journal of Agricultural Economics, 60(1), 48-58, 1978. doi: 10.2307/1240160

Mankiw, N. G. Introdução à economia. $6^{\mathrm{a}}$ ed. Norte Americana, Cengage Learning, 2014. 510p.

Martins, L.; Gouveia e Silva, Z. P.; Silveira, B. C. Produção e comercialização da castanha do brasil (Bertholletia excelsa, h.b.k) no estado do acre- brasil, 1998-2006. Rio Branco Acre. In: Sociedade Brasileira de Economia, Administração e Sociologia Rural (SOBER.), 1-14, 2008.

Muller, C. H.; Fiqueiredo, F. J. C.; Kato, A. K.; Carvalho, J. E. U.; Stein, R. L. B.; Silva, A. B. Castanha-do-Brasil.
Brasília: Embrapa-SPI, 1995. 65 p. (Coleção plantar).

Nogueira, A. K. M.; Santana, A. C.; Garcia, W. S. A dinâmica do mercado de açaí fruto no estado do Pará: de 1994 a 2009. Revista Ceres, 60, 324-331, 2013. Disponível em:http://www.scielo.br/scielo.php?pi$\mathrm{d}=$ S0034737X2013000300004\&script=sci_abstract\&tl$\mathrm{ng}=\mathrm{pt}$.

Nunes, F. S. M. de.; Soares Filho, B. S.; Rodrigues, H. Valorando a floresta em pé: a rentabilidade da castanha do brasil no Acre. In: IX Encontro nacional da economia ecológica Brasília - DF - Brasil, 1-21, 2011. Disponível em: http:// www.ecoeco.org.br/conteudo/publicacoes/encontros/ix_en/ GT1-191-262-20110620235708.pdf.

Peterson, W. L. Returns to poultry research in the United States. Journal of Farm Economics, 49(3), 656-670, 1967.

Randall, A.; Stoll, J. R. Consumer's surplus in commodity space. The American Economic Review, 70(3), 449-455, 1980.

Rathcke, B.; Lacey, E. P. Phenological patterns of terrestrial plants. Annual Review of Ecology and Systematics, 16, 179-214, 1985.

Salomão, R. P. Densidade, estrutura e distribuição espacial de castanheira-do-brasil (Bertholletia excelsa H. \& B.) em dois platôs de floresta ombrófila densa na Amazônia setentrional brasileira. Boletim do Museu Paraense Emílio Goeldi, Ciências Naturais, 4(1),11-25, 2009. Disponível em: http://scielo.iec.pa.gov.br/pdf/bmpegcn/v4n1/v4n1a02.pdf.

Salomão, R. P. A castanheira: história natural e importância econômica. Boletim do Museu Paraense Emílio Goeldi, Ciências Naturais, 9(2), 259-266, 2014. Disponível em: http://repositorio.museu-goeldi.br/handle/mgoeldi/874.

Santana, A. C. de. Métodos quantitativos em economia: elementos e aplicações: Belém: UFRA, 2003. 484p.

Santana, A. C. Elementos de economia, agronegócio e desenvolvimento local. Belém, PA: UFRA, 2005. p.197.

Santana, A. C. de. Valoração de produtos florestais não madeireiros da Amazônia: o caso da castanha-do-brasil. Tese (professor titular) - Universidade Federal Rural da Amazônia (UFRA) - Belém, 2015, 103p. 
Santana, A. C. de; Khan, A. S. Custo social da depredação florestal no Pará: o caso da castanha-do-brasil. Revista de Economia e Sociologia Rural, 30(3), 253-269, 1992.

Santana, A. C. de; Santana, A. L.; Santos, M. A. S. Influência do desmatamento no mercado de madeira em tora da região Mamuru-Arapiuns, Sudoeste do Pará. Revista de Ciências Agrárias, 54(1), 44-53, 2011. Disponível em: https://periodicos.ufra.edu.br/index.php?journal=ajaes\&page $=$ article \&op $=$ view\&path $\% 5 \mathrm{~B} \% 5 \mathrm{D}=281$

Santana, A. C. de; Santana, Á. L; Santana, Á. L.; Martins, C. M. Valoração e sustentabilidade da castanha-do-brasil na Amazônia. Revista de Ciências Agrárias, 60, 77-89, 2017. Disponível em: http://periodicos.ufra.edu.br/index. php/ajaes/article/view/2690.

Santana, A. C. de; Santos, M. A. S. O mercado de caupi no Estado do Pará: aplicação do método dos momentos generalizados. Revista de Ciências Agrárias, 44, 47-58, 2000. Disponível em: https://periodicos.ufra.edu.br/index.php?journal=ajaes\&page $=$ article\&op $=$ view\&path $\% 5 B \% 5 \mathrm{D}=1959$

Santana, A. C. de; Santos, M. A. S.; Santana, Á. L.; Yared, J. A. G. O valor econômico da extração manejada de madeira no Baixo Amazonas, estado do Pará. Revista Árvore, 36, 527-536, 2012. doi: 10.1590/S0100-67622012000300015.

Silva, A. A.; Santos, M. K. V.; Gama, J. R. V.; Noce, R.; Leão, S. Potencial do Extrativismo da Castanha-do-Pará na Geração de Renda em Comunidades da Mesorregião Baixo Amazonas, Pará. Floresta e Ambiente, 20(4), 500509, 2013. Disponível em :http://www.scielo.br/scielo. php?pid=S2179-80872013000400008\&script=sci_abstract\&tlng=pt.
Tonini, H. Fenologia da castanheira-do-brasil (BertholletiaexcelsaHumb. \& Bonpl., Lecythidaceae) no sul do estado de Roraima. Cerne, 17(1), 123-131, 2011. Disponível em: http://www.scielo.br/scielo.php?pi$\mathrm{d}=$ S0104-77602011000100015\&script $=$ sci_abstract\&tl$\mathrm{ng}=\mathrm{pt}$.

Tonini, H., Lopes, C. E. V.; Borges, R. A.; Kaminski, P. E.; Alves, M. S. de.; Fagundes, P. R. O. de. Fenologia, estrutura e produção de sementes em castanhais nativos de Roraima e características socioeconômicas dos extrativistas. Boletim do Museu Paraense Emílio Goeldi. Ciências Naturais, 9(2), 399-414, 2014.

Tonini, H.; Pedrozo, C. . Variações anuais na produção de frutos e sementes de castanheira-do-brasil (Bertholletia excelsa Bonpl., Lecythidaceae) em florestas nativas de Roraima. Revista Árvore, 38(1), 133-144, 2014. doi: 10.1590/ S0100-67622014000100013

Varian, H. R. Microeconomia: uma abordagem moderna. Rio de Janeiro: Elsevier Campus, $9^{\mathrm{a}}$ ed. 2015. 840p. 